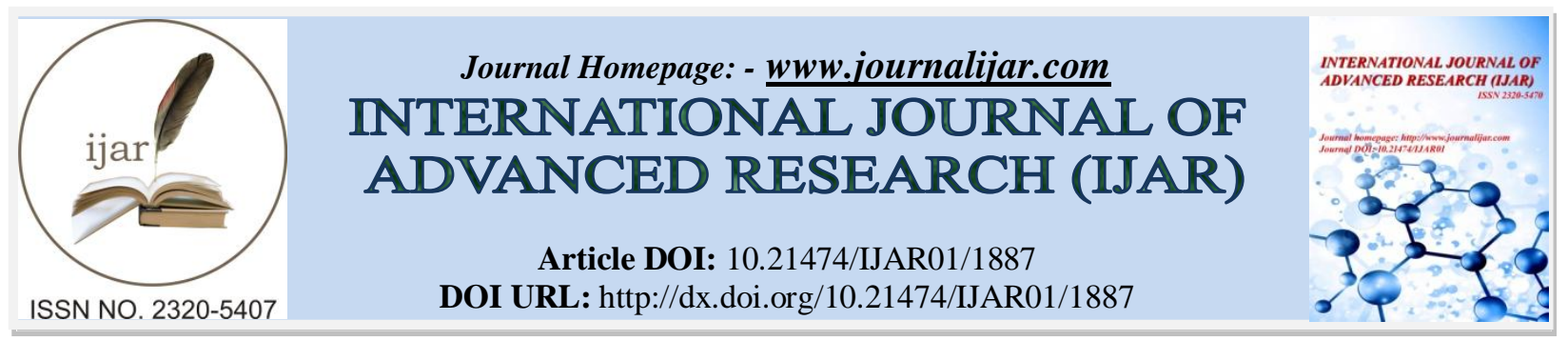

RESEARCH ARTICLE

\title{
POSTMENOPAUSAL BLEEDING: THE VALUE OF TRANS-VAGINAL SONOGRAPHY VERSUS SALINE INFUSION SONO-HYSTEROGRAPHY IN IDENTIFYING THE UTERINE CAUSES FOR BLEEDING.
}

\author{
Dr. Amal HusseinTayah (M.B.Ch.B, DMRD) ${ }^{1}$, Dr. Enas Adnan (F.I.B.M., C.A.B.S/G.O) ${ }^{2}$, Dr. Suha Hussien \\ Hayyawi (F.I.B.M. , C.A.B.S/G.O) ${ }^{3}$, Dr.Takwa Nihad Mohammad (C.A.B.S/G.O) ${ }^{3}$ and Dr. Wasan Ismail Al- \\ Saadi (F.I.B.M.S / radiology $)^{4^{*}}$. \\ 1. Radiology department, Al-Immamain Al-Kadhimain Medical City. \\ 2. Gynaecology \& Obstetric department, College of Medicine, Al-Nahrain University. \\ 3. Gynaecology \& Obstetric department Al-Immamain Al-Kadhimain Medical City. \\ 4. Radiology unit, Surgery department, College of Medicine, Al-Nahrain University.
}

\section{Manuscript Info}

Manuscript History

Received: 12 August 2016

Final Accepted: 16 September 2016

Published: October 2016

Key words:-

postmenopausal bleeding, saline infusion sonohysterography, trans-vaginal sonography .

\section{Abstract}

Background:- Postmenopausal bleeding (PMB) is relatively common problem and a cause for concern due to increased incidence of endometrial carcinoma in this age group

Objective:- To evaluate the diagnostic accuracy of transvaginal sonography (TVS) and saline infusion sonohysterography (SISH) in the assessment of endometrium state in patients with PMB

Methods:- prospective study included 37 patients with PMB. All the patients underwent TVS \& SISH, the endometrium was assessed for maximum thickness, regularity, echogeneity, homogeneity, the presence of mass.The final diagnoses was obtained from histopathology .

Results:- according to the histopathology 16 patients(34.24)\% had normal endometrium , 6 patients(16.22)\% had benign endometrial polyp,12 patients(32.43)\% had endometrial hyperplasia ,2 patients (5.41)\% had submucosal fibroid and 1of the patients (2.70)\% had endometrial carcinoma. Both TVS and SISH were $100 \%$ accurate in making the diagnosis of endometrial Polyp, sub mucosal fibroid and endometrial carcinoma however, TVS overestimated 4 cases of normal endometrium as endometrial hyperplasia giving an accuracy of $89.19 \%$, on the other hand SISH accurately defined all the cases of endometrial hyperplasia

Conclusion:- Both TVS and SISH have comparable accuracy in the assessment of endometrial pathology in women with PMB, however TVS may overestimate endometrial hyperplasia

Copy Right, IJAR, 2016,. All rights reserved.

\section{Introduction:-}

Postmenopausal bleeding (PMB) is a common problem representing 5\% of all gynecological out patients attendance (Moodley and, Robert, 2004) and it is always a cause for concern due to increased percentage of endometrial carcinoma in this age group. Although the most common cause is vaginal atrophy (Munot and Lane, 
2008 ), it is generally accepted that PMB should be suspected as malignant, until proved otherwise(National Institute For Health And Care Excellence Clinical Guideline, 2005) . Several etiological factors may be responsible for PMB, in addition to vaginal atrophy as the use of hormone replacement therapy (HRT), endometrial hyperplasia whether simple or complex (Harding, 2014), or , endometrial cancer (with estimated probability of a woman presenting with PMB to have endometrial cancer is 10-15\%.) (Escoffery et al , 2002 ; Lin et al , 1993) , endometrial polyps (with estimated prevalence of endometrial polyps in patients with PMB and increased endometrial thickness measured with trans-vaginal sonography (TVS) of around 40\%( Epstein et al ,2001 b ; Timmermans et al 2008) ,cervical cancer , Ovarian cancer, especially estrogen-secreting (theca cell) and granulose ovarian tumors(Harding, 2014) . Imaging plays vital role in the assessment of patients with PMB after excluding other clinically obvious causes related to the vagina, vulva or uterine cervix .The choice of imaging is determined according to the appropriateness criteria set by the American college of radiologists (American College of Radiology . ACR Appropriateness Criteria , 2014) . The guidelines are addressing PMB and therefore aimed at excluding cervical cancer, endometrial carcinoma or precancerous lesions of the endometrium(Epstein , 2004 a ; Munro , 2014). According to these guidelines TVS, saline infusion sonohysterography (SISH) and trans-abdominal ultrasound, are the first line imaging modalities in the assessment and follow up of patients with PMB.

We aimed at this study to evaluate the diagnostic accuracy of Trans-vaginal sonography and Saline infusion sonohysterography in the assessment of endometrium state in patients with post menopausal bleeding in correlation with histopathological diagnosis

\section{Patients and methods:-}

This was a prospective study conducted at ultrasound unit at AL-Immamain AL-Kadhimain medical city included patients with post menopausal bleeding who were referred from the gynecology department to investigate the cause of bleeding by TVS and SISH , for the period from the September 2014 to September 2015.The study was approved by the research ethical committee at Al-Nahrain College of Medicine . A verbal consent was obtained from each patient after explaining the details of the procedure. The exclusion criteria were: Patients at active stage of heavy vaginal bleeding, pelvic inflammatory disease(according to the diagnosis made by the gynecologist), Cervical stenosis, Virgin patient ,Previous uterine intervention for PMB like endometrial biopsy or curettage.

The demographic criteria, relevant clinical history and detailed menstrual history were recorded. After performing TVS and SISH at the ultrasound unit, the patients were followed up and those patients for whom histopathology proof of the final diagnosis was obtained, were included in the study

\section{TVS and SISH Protocol:-}

Trans-Vaginal ultrasound examination was performed using multi frequency transvaginal transducer (IC5-9-D ) (Voluson E6 GE Healthcare Austria ). The examination was commenced after emptying the urinary bladder. The TVS probe was covered with sterile condom after filling the tip of the condom with coupling gel , then the probe was introduced into the vagina and the images were obtained in two planes, the endometrium was assessed for maximum thickness, regularity, echogeneity, homogeneity, the presence of mass (size , texture , regularity of the margin , whether the endometrium is draped over the mass or the latter forms an acute angle with the endometrium and the multiplicity) . After documentation of these findings at conventional TVS, the vaginal probe was withdrawn, a brief vaginal examination was performed to aid in locating the cervix. A sterile speculum is placed into the vagina, and the cervix is brought into view. The cervix is then cleansed with povidone -iodine solution. A Foley's catheter of 12 -14Fr size placed at external cervical os using sponge forceps to introduce the catheter inside the uterine cavity, $4 \mathrm{ml}$ of sterile saline used for inflating the balloon of catheter to prevent the catheter from dislodgement , later on , the speculum was removed gently and the balloon is slightly retracted to close the internal cervical os then the TV probe was introduced again through the vagina. Continuous scanning was made in two orthogonal planes while injecting the normal saline. The amount of saline injected ranged from $(10-20 \mathrm{ml})$. After that the catheter was removed and the patient was reminded about the possible increase in intensity of vaginal bleeding and pelvic pain and was reassured that these symptoms are transient and minor. All the patients consulted their referring gynecologists for further management .

The parameters assessed at TVS were re-assessed and recorded after SISH. An endometrial thickness $=$ or $<5 \mathrm{~mm}$, that is of regular homogeneous distinct outlines with central echogenic line within the uterus was deemed normal. On the other hand, if the endometrium measured thicker than $5 \mathrm{~mm}$, it was considered as endometrial hyperplasia. The presence of focally thickened irregular endometrium and/or irregular deformed endometrial lining were 
considered endometrial carcinoma .An entire echogenic focal mass within the endometrial cavity was considered as endometrial polyp. Whereas, if the mass is isoechoic or hypo echoic in comparison to the myometrium and bears a definite relation with myometrium and is reaching the uterine cavity by pushing the endometrium then it is considered as sub mucosal fibroid. These findings were defined according to criteria published by Parsons and Lense(Parsons and Lense, 1993) .

The final diagnoses was obtained from histopathology in (37) patients through endometrial curettage and hysterectomy .

\section{Statistical Analysis:}

The diagnostic accuracy of TVS and SISH were compared using (paired test, fisher exact test, chi square test ). A $P$ value of $<0.05$ was considered statistically significant. These sensitivity, specificity and diagnostic accuracy of TVS and SISH were calculated by comparing the results of both modalities with those obtained by histological examination.

\section{Results:-}

A total number of 37 PMB patients were enrolled in this study, the age range was 51-68years old (mean $57.65 \pm 5.39$ years ) with menopausal duration of 1-20 years (mean $6.3 \pm 4.970$ years ).

The histopathological results of the specimens obtained at surgical intervention revealed that $16 / 37(43.24) \%$ had normal endometrium , 6/37 (16.22) \% had benign polyp ,12/37 (32.43) \% had endometrial hyperplasia , 2/37 (5.41) $\%$ had sub mucosal fibroid and $1 / 37(2.70) \%$ had endometrial carcinoma

AT TVS , 16 patients were found to have endometrial hyperplasia with endometrial thickness rang of (4-17mm, mean $=8.19 \pm 3.46 \mathrm{~mm}$ ) comparing these results with that of histopathology only12 patients were found to have hyperplasia with other 4 found to have normal endometrium, indicating an accuracy of $89.19 \%$ for TVS for the diagnosis of endometrial hyperplasia, on the other hand SISH demonstrated that 12 of the patients were found to have endometrial hyperplasia with endometrial thickness ranging from $3-15 \mathrm{~mm}$ ( mean $=6.97 \pm 3.06 \mathrm{~mm}$ ) , comparing these results with that of histopathology , SISH was found to have an accuracy of $100 \%$ for the diagnosis of endometrial hyperplasia. However both modalities showed comparable high accuracy for the diagnosis of endometrial polyps (table 1)

Comparing the endometrial thickness estimation between the two modalities, TVS gave higher estimation for the endometrial thickness than SISH ( mean endometrial thickness in TVS $=(8.19 \pm 3.46 \mathrm{~mm})$ and in SISH $=(6.97 \pm 3.04$ $\mathrm{mm})$.

No significant statistical differences was found between the TVS and SISH in their ability in differentiating normal from abnormal endometrium ( $p$ value $=0.4725)$ (table 2$)$.

For the two cases of sub mucosal fibroids, both TVS and SISH were able to make confident diagnosis, as well as for the one case with endometrial carcinoma.

The sensitivity ,specificity and accuracy for each modality ( TVS and SISH) for each specific pathology are shown in table 3

Table 1:- the TVS and SISH findings in patients with PMB

\begin{tabular}{|l|l|l|}
\hline \multirow{2}{*}{ FINDINGS } & TVS & SISH \\
\cline { 2 - 3 } & NO. $(\boldsymbol{\%})$ & NO. $(\boldsymbol{\%})$ \\
\hline Normal endometrium & $12(32.43)$ & $16(43.24)$ \\
\hline Polyp & $6(16.22)$ & $6(16.22)$ \\
\hline Endometrial hyperplasia & $16(43.24)$ & $12(32.43)$ \\
\hline sub mucosal fibroid & $2(5.41)$ & $2(5.41)$ \\
\hline Endometrial carcinoma & $1(2.70)$ & $1(2.70)$ \\
\hline
\end{tabular}


Table 2:- Comparison between TVS and SISH defining normal from abnormal state according to histopathological diagnosis

\begin{tabular}{|l|l|l|l|l|l|}
\hline \multirow{2}{*}{ Finding } & TVS & SIS & \multirow{2}{*}{ P value } \\
\cline { 2 - 5 } & No. & \% & No. & \% & \\
\hline Normal & 12 & 32.43 & 16 & 43.24 & 0.4725 \\
\hline Abnormal & 25 & 67.57 & 21 & 56.76 & \\
\hline
\end{tabular}

Table 3:- Sensitivity , specificity and accuracy of TVS and SISH in comparison to histopathological findings.

\begin{tabular}{|l|l|l|l|}
\hline Finding & & TVS & SISH \\
\hline \multirow{5}{*}{ Normal } & Sensitivity & $75 \%$ & $100 \%$ \\
\cline { 2 - 4 } & Specificity & $100 \%$ & $100 \%$ \\
\cline { 2 - 4 } & Accuracy & $89.19 \%$ & $100 \%$ \\
\hline \multirow{3}{*}{ Polyp } & Sensitivity & $100 \%$ & $100 \%$ \\
\cline { 2 - 4 } & Specificity & $100 \%$ & $100 \%$ \\
\cline { 2 - 4 } & Accuracy & $100 \%$ & $100 \%$ \\
\hline Endometrial hyperplasia & Sensitivity & $100 \%$ & $100 \%$ \\
\cline { 2 - 4 } & Specificity & $84 \%$ & $100 \%$ \\
\cline { 2 - 4 } & Accuracy & $89.19 \%$ & $100 \%$ \\
\hline
\end{tabular}

\section{Discussion:-}

The present study evaluated the diagnostic accuracy of TVS and SISH for the detection of endometrial abnormalities in patients with PMB . The evaluation and management of post menopausal bleeding is evolving continuously as the endometrium is more difficult to measure in postmenopausal patients due to relatively poorly defined endomyometrial border. Various techniques can be used in the diagnostic work up of women with postmenopausal bleeding. Diagnostic hysteroscopy can detect focal lesions in the uterine cavity, and a preliminary histological diagnosis can be made(Widrich et al , 1996 ; Schwrzler et al 1998). At conventional TVS examination focal lesions in the uterine cavity may be suspected(Widrich et al , 1996 ; Schwrzler et al 1998; Baldwin et al , 1999; Atri et al , 1994; Williams and Marshburn , 1998), but confirmation by SISH, which involves instillation of saline into the uterine cavity during scanning, improves the accuracy(Widrich et al , 1996; Williams and Marshburn , 1998 ; Gaucherand et al , 1995 ).

In the current study, TVS showed high sensitivity and specificity in the assessment of endometrial pathologies . Various studies have demonstrated wide range of sensitivity and specificity of TVS in assessment of abnormal uterine bleeding in pre and post menopausal women with a sensitivity range from $24-96 \%$ and specificity range from 29-93\% (Ryu et al , 2004; Bree , 1997; Aslam et al , 2007 ; Lalchandani and Phillips , 2003).On the other hand SISH showed even higher sensitivity and specificity in this study, a result that is in agreement with other several studies who reported a sensitivity and specificity of SISH as high as $85-91 \%$ and $83-100 \%$, respectively.( Ryu et al , 2004; O'Connell et al , 1998; Alborzi et al , 2007;Wongsawaeng , 2005) . In a national study (Murad and Ahmed , 2012), they have found a comparable sensitivity for both modalities however SISH was much more specific with a specificity approaching $100 \%$ against $50 \%$ for TVS. What is surprising in the current study is the higher diagnostic performance of TVS in comparison with other studies, this can be attributed to several factors as the limited sample size and the exclusion of the cases with difficult SISH procedure from the study, in addition most of the cases encountered had either normal endometrium with limited cases of endometrial pathologies thus making the task of testing TVS capabilities in diagnosing different endometrial pathologies rather difficult. Comparing both modalities (TVS Vs. SISH) in the current study, TVS over estimated the endometrial thickness in 4 patients and gave false positive results of endometrial hyperplasia (figure1) while SISH correctly identified those 4 patients as having normal endometrium This false positive result obtained by TVS can be explained by the presence of blood clots within the endometrial cavity with similar echo texture to that of the endometrium so that the distinction was not possible at TVS while the instillation of saline at SISH allowed the true discrimination of the endometrium from the blood clots which appear freely mobile and avascular with no clear site of attachment to the endometrium.

the Great majority of women with pathological cavities were defined by SISH and it also can distinguish malignant and various benign conditions, so SISH after TVS allows for identification of focal abnormalities within the 
endometrial cavity, which may then guide biopsy or resection(Jorizzo et al , 1999). This study demonstrated that both TVS and SISH have comparable accuracy for the diagnosis of endometrial polyp , sub mucosal fibroid endomatrial carcinoma and consequently this would help in selecting the patients candidates for other interventional procedure. Several studies have dealt with value of SISH in both pre- and postmenopausal women, Soguktas et al (Soguktas et al , 2012) suggested that SISH can replace hysteroscopy as a method of diagnosing focal lesions in the uterine cavity with high accuracy. Taking into consideration these high diagnostic accuracies, in addition to the wide availability and reproducibility, the low coast and relatively painless procedures, both modalities are when used initially in patients with PMB may obviates the need for further sophisticated or invasive procedures.

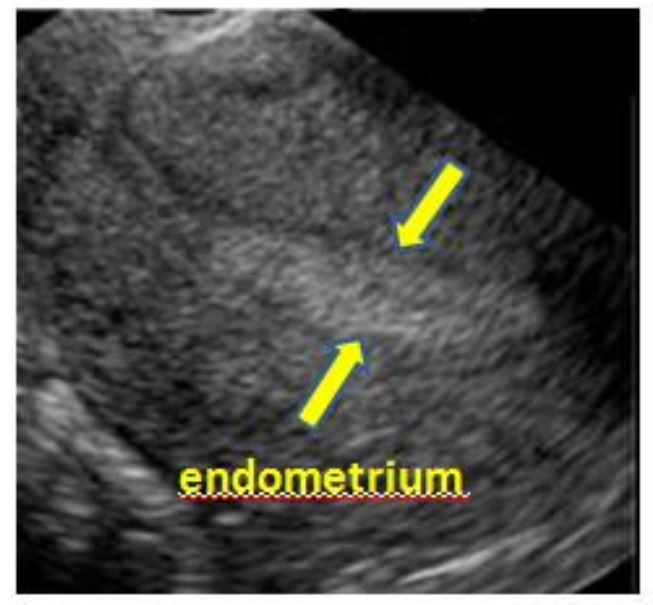

a

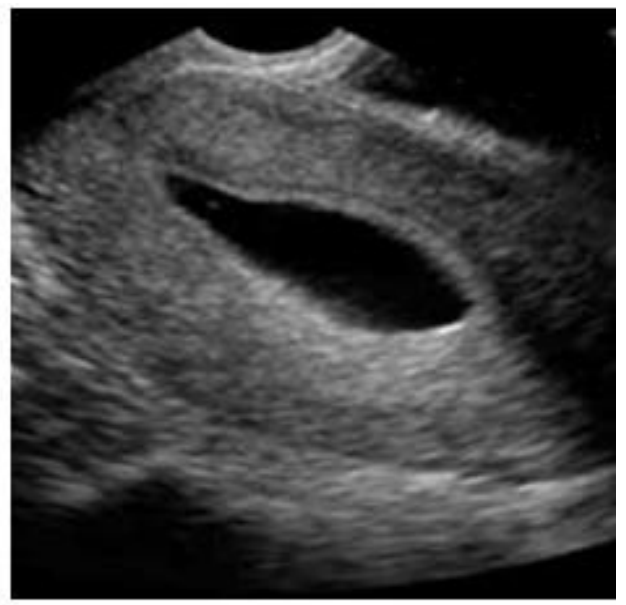

b

Figure 1:- 55 years old patient presented with PMB . (a) TVS reveals thickened endometrium .(b) SISH reveals thin and regular endometrium.

\section{Conclusion:-}

TVS and SISh have comparable highly diagnostic accuracy for evaluation of most focal endometrial pathology but when it comes to endometrial thickening, SISH is more accurate at deciding whether its genuine hyperplasia or merely intra-cavitary clots adding more thickness to the endometrium. SISH can be recommended in patient with post menopausal bleeding when TVS shows endometrial hyperplasia or when the result of TVS are equivocal.

\section{References:-}

1. Alborzi S, Parsanezhad ME, Mahmoodian N, et al . Sonohysterography versus transvaginal sonography for screening of patients with abnormal uterine bleeding Internat. J Gynecol Obstet. 2007;96:20-3.

2. American College of Radiology . ACR Appropriateness Criteriaß. Clinical Condition: Abnormal Vaginal Bleeding , revised 2014

3. Aslam M, Ijaz L, Tariq SH, et al. Comparison of Transvaginal Sonography and Saline Contrast Sonohysterography in Women with Abnormal Uterine Bleeding: Correlation with Hysteroscopy and Histopathology Int J Health Sci (Qassim). 2007 Jan; 1(1): 17-24

4. Atri M, Nazarnia S, Aldis AE, et al .Transvaginal US appearance of endometrial abnormalities. Radiographics 1994; 14: 483-92

5. Baldwin MT, Dudiak KM, Gorman B, et al . Focal intracavitary masses recognized with the hyperechoic line sign at endovaginal US and characterized with hysterosonography. Radiographics 1999; 19: 927-35

6. Bree RL. Ultrasound of the endometrium: facts, controversies, and future trends. Abdom Imaging. 1997;22:557-68.

7. Epstein E., "Management of postmenopausal bleeding inSweden: a need for increased use of hydrosonography and hysteroscopy,” Acta Obstetricia et Gynecologica Scandinavica,vol. 83, no. 1, pp. 89-95, 2004. 
8. Epstein E., Ramirez A., Skoog L, et al .Dilatation and curettage fails to detect most focal lesions in the uterine cavity in women with postmenopausal bleeding. Acta Obstetricia et Gynecologica Scandinavica, vol. 80, no. 12, pp. 1131-1136, 2001.

9. Escoffery T., G. O. Blake, and L. A. Sargeant, "Histopathological findings in women with postmenopausal bleeding in Jamaica," West Indian Medical Journal, vol. 51, no. 4, pp. 232-235, 2002.

10. Gaucherand P, Piacenza JM, Salle B, et al . Sonohysterography of the uterine cavity: preliminary investigations. J Clin Ultrasound 1995; 23: 339-48

11. Harding M. Postmenopausal bleeding. At http://patient.info/doctor/postmenopausal-bleeding .2014

12. Jorizzo JR, Riccio GJ, Chen MY, et al.Sonohysterography: the next step in the evaluation of the abnormal endometrium. Radiographics 1999;19:S117-S130.

13. Lalchandani S, Phillips K. Evaluation of endometrial cavity-investigation options. Rev Gynaecol Prac. 2003;3:165-70.

14. Lin H, Wu M., Shyu M.,et al, "Clinical study of 381 postmenopausal bleedingpatients,” Journal of the Formosan Medical Association, vol. 92,no. 3, pp. 241-244, 1993.

15. Moodley M, Roberts C; Clinical pathway for the evaluation of postmenopausal bleeding with an emphasis on endometrial cancer detection. J Obstet Gynaecol. 2004 Oct;24(7):736-41

16. Munot S, Lane G: Modern management of post menopausal bleeding. Trends in Urology, Gynaecology and Sexual health. Vol 13. Issue 5. Sept 2008)

17. Munro M, The Southern California Permanente Medical Group's Abnormal Uterine Bleeding Working group investigation of Women with Postmenopausal Uterine Bleeding: Clinical Practice Recommendations Volume 18(1); Winter 2014

18. Murad A. Ahmed F. Saline infusion sonohysterography versus transvaginal sonography in the assessment of post menopausal bleeding. Iraqi Medical Journal . 2012 ;58(2):138-144

19. National Institute For Health And Care Excellence Clinical Guideline :Referral for suspected cancer . June 2005

20. O'Connell LP, Fries MH, Zeringue E, et al . Triage of abnormal postmenopausal bleeding: a comparison of endometrial biopsy and transvaginal sonohysterography versus fractional curettage with hysteroscopy. Am $\mathbf{J}$ Obstet Gynecol. 1998;178:956-61.

21. Parsons AK, Lense JJ. Sonohysterography for endometrial abnormalities: preliminary results. J Clin Ultrasound 1993; 21:87-95.

22. Ryu J, Kim B, Lee J, et al .Comparison of transvaginal ultrasonography with hysterosonography as a screening method in patients with abnormal uterine bleeding. Korean J Radiol. 2004;5:39-46.

23. Schwrzler P, Concin H, Bosch H, et al . An evaluation of sonohysterography and diagnostic hysteroscopy for the assessment of intrauterine pathology. Ultrasound Obstet Gynecol 1998; 11: 337-42

24. Soguktas S., Cogendez E., Kayatas S., et al. Comparison of saline infusion sonohysterography and hysteroscopy in diagnosis of premenopausal women with abnormal uterine bleeding. European Journal of Obstetrics and Gynecology and Reproductive Biology161 (2012) 66-70

25. Timmermans A., Gerritse M. B., B.. Opmeer C, et al. "Diagnostic accuracy of endometrial thickness to exclude polyps in women with postmenopausal bleeding," Journal of Clinical Ultrasound, vol. 36, no. 5, pp.286-290, 2008

26. Widrich T, Bradley LD, Mitchinson AR, Collins RL. Comparison of saline infusion sonography with office hysteroscopy for the evaluation of the endometrium. Am J Obstet Gynecol 1996; 174: 1327-34

27. Williams CD, Marshburn PB. A prospective study of transvaginal hydrosonography in the evaluation of abnormal uterine bleeding. Am J Obstet Gynecol 1998; 179: 292-8

28. Wongsawaeng W. Transvaginal ultrasonography, sonohysterography and hysteroscopy for intrauterine pathology in patients with abnormal uterine bleeding. J Med Assoc Thai. 2005;88:77-81. 